\title{
Notbremsen
}

\author{
Karl Rihaczek
}

Liebe Leserinnen, liebe Leser!

Mit der Notbremse soll man eine unmittelbar drohende Gefahr abwehren. Man bringt den Zug zum Stehen, in der Hoffnung, dass die Abwehr danach leichter fällt. Das wird damit erkauft, dass man dabei strandet. Wenn man damit Leben retten konnte, war es das wert gewesen. Aber Ärger bereitet es allemal. So überlegt man es sich auch im Ernstfall zweimal, bevor man die Notbremse zieht.

In Amerika sorgt man sich um das Behavioral Targeting. Gewisse Firmen aus der Werbebranche beobachten einerseits per Browser Cookie, für welche Anzeigen der Teilnehmer Interesse zeigt, und platzieren andererseits Anzeigen ihrer Auftraggeber auf seine Web-Seite. Jedes Anklicken von Werbebannern wird registriert. So entsteht ein Interessen-Profil des Teilnehmers. Damit kann der Behavioral-Targeting-Betreiber dem Teilnehmer die ihn interessierende Werbung einblenden und so bei der Platzierung von Anzeigen eine bessere Trefferquote erzielen. Und er belästigt den Teilnehmer nicht unnötig. Dessen Identität wird dazu nicht gebraucht. Was ihm gezeigt wird, ergibt sich allein aus seinem Klick-Verhalten. Er kann anonym bleiben. Allerdings wird er für die Werber auch als Anonymer mit der Zeit zunehmend transparent und damit auch eventuell individualisierbar. Da$\mathrm{zu}$ ist ferner zu beachten, dass Government Officials und Zivilprozessparteien mit einfacher richterlicher Genehmigung $\mathrm{Zu}$ griff auf die Verhaltensprofile zugestanden wird. Sie sind durchaus in der Lage, einen Personenbezug herzustellen.

Bereits 1999 wollte DoubleClick, ein Behavioral-Targeting-Anbieter, sein Angebot verbessern und sein online Gesammeltes durch offline Gesammeltes anreichern. DoubleClick wollte darum mit Abacus, einer Market-Research-Firma, fusionieren. Aber dagegen erhob sich öffentlicher Protest. Die beiden Firmen sahen daraufhin von der Fusion ab; die Branche setzte sich mit

${ }^{1}$ Siehe „David McGuire“ dmcguire@cdt.org 08.08.2007, Policy Post 13.11: Privacy Initiatives Key to Addressing Behavioral Targeting der Federal Trade Commission FTC zusammen und beschloss eine Network Advertising Initiative NAI. Mit ihr verpflichteten sich ihre Unterzeichner auf die Einhaltung von festgelegten Privacy Rules. Dazu gehört auch die strikte Trennung von online- und offline-Verhaltensprofilen. Das Abkommen hielt einige Jahre lang.

Inzwischen ist einiges an Technik dazu gekommen: Internet, Rundfunk-, Kabelund Telefon-Dienste rücken näher zusammen. Adware-Firmen, deren Programme separat vom Browser installiert sind, können das gesamte Browsing-Verhalten des Teilnehmers beobachten. Auch InternetSuchdienste und E-Mail-Speicherdienste können ihres beitragen. Ob solcher Aussichten schwinden die Hemmungen in bezug auf Privacy-Verletzungen. Man will das technische Angebot aktivieren und ein verbessertes Verhaltensprofil des Teilnehmers anbieten können. Dazu plant eine Reihe von Firmen Fusionen oder Aufkäufe: Google und Double Click, Yahoo und RightMedia, Microsoft und aQuantive, die WPPGruppe und 24/7 RealMedia, AOL und Tacoda. Die Angebote sollen vernetzt, die Verhaltensprofile geschärft, die geschärften Profile auf die vereinigten Service-Bereiche angewendet werden. Damit wird der NAI Grundsatz der Trennung von online und offline fallen gelassen. Die Werber streben Synergie ihrer Anstrengungen an. Sie wollen sich den Teilnehmer transparent machen können. Sollte dazu seine Einwilligung erforderlich sein, ist das kein Problem, verstehen sie sich doch professionell darauf, Menschen passend zu beeinflussen. Dem Recht auf Privacy im Internet lässt sich der Stachel nehmen.

So fragt man sich in Amerika, ob sich unter diesen Umständen der Gesetzgeber mit der NAI weiterhin zufrieden geben darf. Die interessierte Öffentlichkeit verlangt eine gesetzliche Regelung. Der Werbebranche wird damit bewusst, dass sie sich mit ihren Vorhaben eventuell auf Glatteis begibt. So beugen die Firmen einer staatlichen Regelung vor und sorgen sich um PrivacyInteressen ihrer Kunden. Sie wollen z.b. unterschiedliche Tools bereit stellen, mit denen etwa die Lebensdauer der Cookies beschränkt werden kann, oder mit denen eventuelle Personenbezüge unkenntlich gemacht werden können. In einem Fall soll der Betroffene selbst die Möglichkeit haben, das Speichern seiner Suchanfragen zu verhindern. Er kann freilich grundsätzlich das Opt-out wählen. Er kann den Werber auffordern, ihn vom Service auszunehmen. Dann wird das Cookie im Browser gelöscht und das Behavioral Targeting eingestellt. Damit verzichtet der Teilnehmer auf die interessantere gezielte Werbung; die lästige ungezielte Werbung bleibt ihm erhalten. Mit Hinweis darauf und mit der brancheneigenen Professionalität beeindruckt der Werber den Teilnehmer und macht ihm das Opt-out zum Problem. Entschließt dieser sich dennoch dazu, zieht er damit die Notbremse, bringt aber den Zug nicht ganz zum Stehen. Er muss bei der Unübersichtlichkeit des Internet befürchten, dass er sich Unannehmlichkeiten eingehandelt hat. Es ist verunsichert.

Über die NAI hinaus auf den Gesetzgeber $\mathrm{zu}$ blicken, dürfte also gerechtfertigt sein. Man will, dass er hier Anwendungen der Technik unter Kontrolle bringt. Dazu müsste er aber auch die Entwicklung der Technik unter Kontrolle haben. Das hat noch kein Gesetzgeber jemals geschafft. Wie auch? Die Situation, liebe Leserinnen und Leser, erinnert mich an 1975 und einen Ministerialbeamten: „Wie, das geht technisch nicht?“", sagte dieser, „Nix da! Lange genug ist das Recht der Technik hinterher gelaufen. Nun (mit dem Datenschutz) werdet ihr euch nach dem Recht richten müssen." Technische Entwicklung umbiegen oder gar verhindern? Sollte der Gesetzgeber notfalls die Notbremse ziehen?

Mit freundlichen Grüßen, Ihr

\section{Karl Rhaorel}

